\title{
Nonlinear Dynamics of Cournot Duopoly Game: When One Firm Considers Social Welfare
}

\author{
S. S. Askar ${ }^{1,2}$ and A. A. Elsadany ${ }^{3}$ \\ ${ }^{1}$ Department of Statistics and Operations Research, College of Science, King Saud University, Riyadh, Saudi Arabia \\ ${ }^{2}$ Department of Mathematics, Faculty of Science, Mansoura University, Mansoura, Egypt \\ ${ }^{3}$ Department of Basic Science, Faculty of Computers and Informatics, Suez Canal University, Ismailia 41522, Egypt
}

Correspondence should be addressed to S. S. Askar; s.e.a.askar@hotmail.co.uk

Received 15 December 2020; Revised 12 January 2021; Accepted 15 January 2021; Published 27 January 2021

Academic Editor: Ya Jia

Copyright (c) 2021 S. S. Askar and A. A. Elsadany. This is an open access article distributed under the Creative Commons Attribution License, which permits unrestricted use, distribution, and reproduction in any medium, provided the original work is properly cited.

\begin{abstract}
In this paper, we study the competition between two firms whose outputs are quantities. The first firm considers maximization of its profit while the second firm considers maximization of its social welfare. Adopting a gradient-based mechanism, we introduce a nonlinear discrete dynamic map which is used to describe the dynamics of this game. For this map, the fixed points are calculated and their stability conditions are analyzed. This includes investigating some attracting set and chaotic behaviors for the complex dynamics of the map. We have also investigated the types of the preimages that characterize the phase plane of the map and conclude that the game's map is noninvertible of type $Z_{4}-Z_{2}$.
\end{abstract}

\section{Introduction}

Because of the appearance of the wealth theory in $[1,2]$, many studies on Cournot and Bertrand games have been raised. For instance, Singh and Vives introduced a quadratic utility function that has been used to model and study a Cournot duopoly game in [3]. This utility function has been also adopted in different games by many authors such as Askar [4-7], Elsadany [8], Naimzada and Tramontana [9], and $\mathrm{Ma}$ and $\mathrm{Pu}[10]$. Modelling such games in a discrete time periods requires some mechanisms such as bounded rationality, Puu's approach, naive expectation, and other adaptive methods.

Several studies have been adopted both bounded rationality and Puu's incomplete information. They are two different mechanisms. For the bounded rationality, the game's players (or firms) are updating their output production depending on discrete time steps and by using a local estimate of their marginal profits. Furthermore, the players do not have to possess a complete knowledge of both demand and cost functions. However, they instead want to discover whether the market responses to small changes in production using an estimation of their marginal profits. For more applications on this mechanism which is sometimes called a myopic mechanism, one can see $[11,12]$. The socalled Puu's mechanism has been introduced in [13]. It is characterized by its realistic feature that is the firms do not have to know the profit function in order to get estimation of the commodity produced at the next period of time. The firms only require the commodity and profit at the past two periods of time. There are some other updating mechanisms that have been reported in the literature. For instance, Long and Huang [14], Agiza and Elsadany [15], Kopel [16], Elabbasy et al. [17, 18], Askar and Abouhawwash [19], Hommes [20], Tremblay et al. [21], Ahmed et al. [22], Baiardi and Naimzada [23], Fanti et al. [24], Elsadany and Awad [25], Tremblay and Tremblay [26], Askar and AlKhedhairi [27], and Gao and Du [28].

The majority of the literature has been analyzed for models of mixed oligopoly on the basis that the game has been in a static case. Liu et al. [29] investigated the static game of endogenous horizontal product differentiation in a mixed duopoly. The relationship between privatization and corporate tax policies has been studied in [30]. Nie [31] has 
analyzed the effects of capacity constraints on the mixed duopoly game. The strategy for cost-reduction innovation in the mixed economy has been explored in [32]. All of earlier papers have not discussed the case of a dynamic mixed oligopoly model.

The current paper introduces a dynamic game of Cournot duopoly on which two firms are competing but they are different in optimization process. The first firm focuses on maximizing its profit that depends on a quadratic production cost. The second firm is different and wants to maximize its social welfare using the same quadratic cost form. The main contribution considers introducing such optimization problem and dynamic characteristics emanated from the game's map. Furthermore, the monopolistic case is studied and is shown that each firm behaves monopolistically such as the standard logistic process. In the duopolistic case, the equilibrium points are calculated and their stability conditions are analyzed. Indeed, this includes local and global analysis of the routes by which these equilibrium points can be destabilized. The numerical simulation shows that some attracting sets are born due to both period-doubling and Neimark-Sacker bifurcations. In addition, the nonlinearity and noninvertibility of the game's map give rise to such complex behaviors.

The outline of current paper is divided into the following sections. After the introduction, we give in Section 2 the description of the game represented by a two-dimensional discrete dynamic map. In Section 3, we calculate the game's fixed points and study their stability. Furthermore, we give in this section a detailed discussion on the monopolistic case. In addition, we study by numerical simulation the attracting sets and chaotic behaviors arise due to the dynamics of the duopoly case. Moreover, we investigate the critical curves of the map and categorize the phase plane regions. Finally, we give our conclusion on the obtained results within this paper.

\section{Cournot Duopoly Game}

The game is constructed based on two competing firms with quantity-based strategies and differentiated products. The quantities produced by the two firms are denoted by $q_{1}$ and $q_{2}$. Their demand functions are obtained by recalling the following utility function:

$$
U\left(q_{1}, q_{2}\right)=a\left(q_{1}+q_{2}\right)-\frac{1}{2}\left(q_{1}^{2}+2 \mathrm{~d} q q_{2}+q_{2}^{2}\right) .
$$

More information on this utility is given in [3]. Both $a$ and $d$ are constants. Supposing the budget constraint $p_{1} q_{1}+p_{2} q_{2}=M$, we get the following maximization problem:

$$
\begin{aligned}
& \operatorname{Max} U\left(q_{1}, q_{2}\right), \\
& \text { s.t. } \quad p_{1} q_{1}+p_{2} q_{2}=M .
\end{aligned}
$$

Solving (2) gives

$$
\left\{\begin{array}{l}
p_{1}=a-q_{1}-\mathrm{d} q_{2} \\
p_{2}=a-q_{2}-\mathrm{d} q_{1}
\end{array}\right.
$$

where $p_{1}$ and $p_{2}$ denote the retail prices of the two firms' products, respectively. The constants $a>0$ represent the maximum price while $d \in[-0.5,1]$ represents the product differentiation. $d=1$ indicates homogeneous products while $d=0$ means we have two monopolistic firms. Consider the following cost function:

$$
C_{i}\left(q_{i}\right)=\frac{c}{2} q_{i}^{2}, \quad i=1,2,
$$

where $c>0$. Now, the profits of the two firms are given by

$$
\begin{aligned}
& \pi_{1}=p_{1} q_{1}-\frac{c}{2} q_{1}^{2}=\left(1-q_{1}-\mathrm{d} q_{2}\right) q_{1}-\frac{c}{2} q_{1}^{2}, \\
& \pi_{2}=\left(1-q_{2}-\mathrm{d} q_{1}\right) q_{2}-\frac{c}{2} q_{2}^{2} .
\end{aligned}
$$

The consumer surplus is assumed to be CS $=(1 / 2)\left(q_{1}^{2}+\right.$ $\left.q_{2}^{2}+2 \mathrm{~d} q_{1} q_{2}\right)$. The social welfare is defined as the sum of consumer surplus and profits as follows: $W=\mathrm{CS}+\pi_{1}+\pi_{2}$. Now, both firms want to maximize the following payoffs:

$$
\begin{aligned}
& \pi_{1}=\left(a-q_{1}-\mathrm{d} q_{2}\right) q_{1}-\frac{c}{2} q_{1}^{2}, \\
& \Pi_{2}=\omega \pi_{2}+(1-\omega) W,
\end{aligned}
$$

where $\omega \in[0,1]$. We should highlight that lack of market demand and consumer information bring difficulty for producers. Therefore, producers estimate the market demand by adopting the gradient mechanism defined below:

$$
\left\{\begin{array}{l}
q_{1}(t+1)=q_{1}(t)+v_{1} q_{1} \frac{\partial \pi_{1}}{\partial q_{1}}, \\
q_{2}(t+1)=q_{2}(t)+v_{2} q_{2} \frac{\partial \Pi_{2}}{\partial q_{2}},
\end{array}\right.
$$

where $v_{i}$ represents the adjustment speed for the $i^{\text {th }}$ firm. Using (6) in (7), we get the map's game:

$$
T\left(q_{1}, q_{2}\right):\left\{\begin{array}{l}
q_{1}(t+1)=q_{1}(t)+v_{1} q_{1}\left[a-(2+c) q_{1}-\mathrm{d} q_{2}\right], \\
q_{2}(t+1)=q_{2}(t)+v_{2} q_{2}\left[a-(1+\omega+c) q_{2}-\mathrm{d} q_{1}\right] .
\end{array}\right.
$$

This map is a quadratic discrete dynamic map, and it is converted into Fanti's map [24] at $\omega=1$.

\section{Equilibrium Points and Their Stability}

Setting $q_{i}(t+1)=q_{i}(t)=q_{i}, \quad i=1,2$ in (8), one obtains

$$
\left\{\begin{array}{l}
q_{1}\left[a-(2+c) q_{1}-\mathrm{d} q_{2}\right]=0, \\
q_{2}\left[a-(1+\omega+c) q_{2}-\mathrm{d} q_{1}\right]=0 .
\end{array}\right.
$$

Solving algebraically system (9), we get four fixed points as follows: 


$$
\begin{aligned}
E_{0}= & (0,0), \\
E_{1}= & \left(\frac{a}{c+2}, 0\right), \\
E_{2}= & \left(0, \frac{a}{c+\omega+1}\right), \\
E_{*}= & \left(q_{1}^{*}, q_{2}^{*}\right) \\
& =\left(\frac{a+a \omega+a c-a d}{3 c+2 \omega+c \omega+c^{2}-d^{2}+2}, \frac{2 a+a c-a d}{3 c+2 \omega+c \omega+c^{2}-d^{2}+2}\right),
\end{aligned}
$$

where $E_{0}, E_{1}, E_{2}$ are called boundary fixed points while $E_{*}$ is called a Nash equilibrium point. It should be noted that the equilibrium points $E_{*}$ become the same as of Fanti's equilibrium point when $\omega=1$. Studying the stability of these points requires to calculate the Jacobian matrix of the map:

$J=\left[\begin{array}{cc}1+v_{1}\left[a-2(2+c) q_{1}-\mathrm{d} q_{2}\right] & -v_{1} \mathrm{~d} q_{1} \\ -v_{2} \mathrm{~d} q_{2} & 1+v_{2}\left[a-2(1+\omega+c) q_{2}-\mathrm{d} q_{1}\right]\end{array}\right]$.

It has the following characteristic polynomial:

$$
f(\lambda)=\lambda^{2}-\operatorname{tr}(J(E)) \lambda+\operatorname{det}(J(E)),
$$

where $\operatorname{tr}(J)$ and $\operatorname{det}(J)$ represent trace and determinant of (11). They are used in Jury conditions [4]. These conditions are given by

$$
\begin{aligned}
f(1) & =1-\operatorname{tr}(J(E))+\operatorname{det}(J(E))>0, \\
f(-1) & =1+\operatorname{tr}(J(E))+\operatorname{det}(J(E))>0, \\
\operatorname{det}(J) & <1 .
\end{aligned}
$$

The above conditions characterize different types of bifurcations by which the equilibrium points may be unstable. These types are summarized in the following:

(1) Period-doubling bifurcation is raised when $f(-1)=0$

(2) Transcritical or fold bifurcation is raised when $f(1)=0$

(3) Neimark-Sacker bifurcation is raised when det $(J)<1$

Now, we study the stability of the fixed points.

Theorem 1. The fixed point $E_{0}$ is unstable point.
Proof. The Jacobian matrix given in (11) at this point becomes

$$
J\left(E_{0}\right)=\left[\begin{array}{cc}
1+\nu_{1} a & 0 \\
0 & 1+\nu_{2} a
\end{array}\right]
$$

It is clear that (15) is a diagonal matrix and hence its eigenvalues become $\lambda_{1}=1+v_{1} a$ and $\lambda_{2}=1+v_{2} a$. Because of the positivity of the parameters $a, v_{1}$, and $v_{2}$, we have $\left|\lambda_{1,2}\right|>1$ and so $E_{0}$ is unstable repelling node.

Theorem 2. The fixed point $E_{1}$ is saddle point.

Proof. The Jacobian matrix given in (11) at this point becomes

$$
J\left(E_{1}\right)=\left[\begin{array}{cc}
1-v_{1} a & \frac{v_{1} d}{c+2} \\
0 & 1+v_{2}\left(\frac{2 a}{c+2}\right)
\end{array}\right] .
$$

It is clear that (15) is a triangular matrix and hence its eigenvalues become $\lambda_{1}=1-\nu_{1} a$ and $\lambda_{2}=1+\nu_{2}(2 a /(c+2))$. It is simple to see $\left|\lambda_{1}\right|<1$ and $\left|\lambda_{2}\right|>1$. Therefore, the fixed point $E_{1}$ is saddle point.

Theorem 3. The fixed point $E_{2}$ is saddle point.

Proof. The Jacobian matrix given in (11) at this point becomes

$$
J\left(E_{2}\right)=\left[\begin{array}{cc}
1+v_{1}\left(\frac{a(c+\omega+1-d)}{c+\omega+1}\right) & 0 \\
\frac{-v_{2} a d}{c+\omega+1} & 1-a v_{2}
\end{array}\right] .
$$

It is clear that (16) is a triangular matrix and hence its eigenvalues $\lambda_{1}=1+\nu_{1}((a(c+\omega+1-d)) /(c+\omega+1)) \quad$ and $\lambda_{2}=1-a \nu_{2}$. Because of the positivity of the parameters $a, v_{1}, v_{2}$, and $d<1$, we have $\left|\lambda_{1}\right|>1$ and $\left|\lambda_{2}\right|<1$. Therefore, the point $E_{2}$ is saddle point.

For Nash equilibrium point, the Jacobian becomes

$$
\mathbf{J}\left(E_{*}\right)=\left(\begin{array}{cc}
1-\frac{a(2+c)(1+\omega+c-d)}{2+2 \omega+3 c-d^{2}+c \omega+c^{2}} v_{1} & -\frac{a d(1+\omega+c-d)}{2+2 \omega+3 c-d^{2}+c \omega+c^{2}} \nu_{1} \\
-\frac{a d(2+c-d)}{2+2 \omega+3 c-d^{2}+c \omega+c^{2}} v_{2} & 1-\frac{a(2+c-d)(1+\omega+c)}{2+2 \omega+3 c-d^{2}+c \omega+c^{2}} v_{2}
\end{array}\right),
$$

whose trace and determinant are given by 


$$
\begin{aligned}
\operatorname{tr}\left(\mathbf{J}\left(E_{*}\right)\right) & =2-\frac{a(2+c)(1+\omega+c-d) v_{1}+a(1+\omega+c)(2+c-d) v_{2}}{2+2 \omega+3 c-d^{2}+c \omega+c^{2}}, \\
\operatorname{Det}\left(\mathbf{J}\left(E_{*}\right)\right) & =1+\frac{a^{2}(2+c-d)(1+\omega+c-d) v_{1} v_{2}-a(1+\omega+c)(2+c-d) v_{2}-2 a(2+c)(1+\omega+c-d) v_{1}}{2+2 \omega+3 c-d^{2}+c \omega+c^{2}}
\end{aligned}
$$

The eigenvalues of $\mathbf{J}\left(E_{*}\right)$ have a long analytical form, and instead we discuss the stability of the Nash equilibrium point by using Jury conditions:

$$
\left\{\begin{array}{l}
1-\operatorname{tr}\left(\mathbf{J}\left(E_{*}\right)\right)+\operatorname{Det}\left(\mathbf{J}\left(E_{*}\right)\right)>0 \\
1+\operatorname{tr}\left(\mathbf{J}\left(E_{*}\right)\right)+\operatorname{Det}\left(\mathbf{J}\left(E_{*}\right)\right)>0 \\
1-\operatorname{Det}\left(\mathbf{J}\left(E_{*}\right)\right)>0
\end{array}\right.
$$

$$
\begin{aligned}
\frac{a^{2}(2+c-d)(1+\omega+c-d) v_{1} \nu_{2}}{2+2 \omega+3 c-d^{2}+c \omega+c^{2}} & >0 \\
4-\Phi & >0 \\
\Phi & >0 \\
\Phi & =\frac{a(1+\omega+c)(2+c-d) v_{2}+2 a(2+c)(1+\omega+c-d) \nu_{1}-a^{2}(2+c-d)(1+\omega+c-d) \nu_{1} \nu_{2}}{2+2 \omega+3 c-d^{2}+c \omega+c^{2}}
\end{aligned}
$$

which can be rewritten in the form

It is easy to see that the first condition of (20) is always fulfilled. If the other two conditions are fulfilled, then $E_{*}$ is locally asymptotically stable provided that $0<\Phi<4$. On the other hand, if $\Phi \geq 4$, this means $E_{*}$ gets unstable due to the coexistence of period-doubling bifurcation. In addition, it becomes unstable due to Neimark-Sacker bifurcation provided that $\Phi \leq 0$. The next section gives some insights about the above analytical analysis.

3.1. Discussion and Numerical Simulation. Let us now discuss the monopoly case of map (8). It is easy to see that this map is trapped to the point $(0,0)$ which means at $q_{1}(t)=0$ or $q_{2}(t)=0$, it gives $q_{1}(t+1)=0$ or $q_{2}(t+1)=0$. Setting $q_{1}(t)=0$ or $q_{2}(t)=0$ in (8), one gets the following:

$$
\begin{aligned}
& q_{1}(t+1)=q_{1}(t)+v_{1} q_{1}(t)\left[a-(2+c) q_{1}(t)\right], \\
& q_{2}(t+1)=q_{2}(t)+v_{2} q_{2}(t)\left[a-(1+\omega+c) q_{2}(t)\right],
\end{aligned}
$$

which can be simplified to

$$
\begin{aligned}
& q_{1}(t+1)=\left(1+a v_{1}\right) q_{1}(t)\left(1-\frac{v_{1}(2+c)}{1+a v_{1}} q_{1}(t)\right) \\
& q_{2}(t+1)=\left(1+a v_{2}\right) q_{2}(t)\left(1-\frac{v_{2}(1+\omega+c)}{1+a v_{2}} q_{2}(t)\right) .
\end{aligned}
$$

Separately, each part of (22) conjugates the standard logistic map, $y_{j}(t+1)=\mu_{j} y_{j}(t)\left(1-y_{j}(t)\right), j=1,2$. Then, we have the following linear transformations for (22): lopenup4

$$
\begin{aligned}
q_{1}(t) & =\frac{1+a v_{1}}{v_{1}(2+c)} y_{1}(t), \\
q_{2}(t) & =\frac{1+a v_{2}}{v_{2}(1+\omega+c)} y_{2}(t), \\
\mu_{j} & =1+a v_{j} ; \quad j=1,2 .
\end{aligned}
$$

This implies that the dynamics of (22) are the same as the logistic map. Each part in (22) is separately a unimodel map. Both have unique critical points $C_{q_{1}}^{-1}$ and $C_{q_{2}}^{-1}$ with coordinates given by

$$
\begin{aligned}
& \breve{q}_{1}=\frac{1+a v_{1}}{2 v_{1}(2+c)}, \\
& \breve{q}_{2}=\frac{1+a v_{2}}{2 v_{2}(1+\omega+c)} .
\end{aligned}
$$

These coordinates conjugate the critical points $y_{1}=(1 / 2)$ and $y_{2}=(1 / 2)$. In addition, system (22) has the following fixed points: 


$$
\begin{aligned}
& q_{1}^{O}=0, \\
& q_{1}^{O_{1}}=\frac{a}{2+c}, \\
& q_{2}^{O}=0, \\
& q_{2}^{O_{2}}=\frac{a}{1+\omega+c},
\end{aligned}
$$

which conjugate $y_{1}=0, y_{1}=1-\left(1 / \mu_{1}\right), y_{2}=0$, and $y_{2}=$ $1-\left(1 / \mu_{2}\right)$ of the logistic map. It is easy to see that $\left|\left(\mathrm{d} q_{i}(t+1)\right) /\left(\mathrm{d} q_{i}(t)\right)\right|_{q_{i}^{O}=0}=1+a v_{i}>1, i=1,2$ and then $q_{i}^{O}, i=1,2$ is an unstable repelling point. In addition, we can see that both $q_{1}^{O_{1}}$ and $q_{2}^{O_{2}}$ are stable attracting points under the condition $0<a v_{i}<2, i=1,2$. At $a v_{i}>2, i=1,2$, the dynamics of each part in (22) may be a period cycle or a cyclic chaotic attractor. These behaviors are characterized by basins of attraction that are bounded by the repelling points $q_{1}^{O}$ or $q_{2}^{O}$ and their preimages. These preimages are obtained by setting $q_{i}(t+1)=0, i=1,2$ in $(22)$ as follows:

$$
\begin{aligned}
& q_{1}^{O_{-1}}=\frac{1+a v_{1}}{v_{1}(2+c)}, \\
& q_{2}^{O_{-1}}=\frac{1+a v_{2}}{v_{2}(1+\omega+c)},
\end{aligned}
$$

which conjugate $y_{1}=1$ and $y_{2}=1$ in the logistic map. Therefore, for the first part of (22), any trajectories starting out of the interval $\left[0, q_{1}^{O_{-1}}\right]$ will be divergent to $-\infty$. The same observation is for the second part of (22). In order to validate the obtained results in the monopoly case, we use numerical simulation by assuming the following parameters' values: $a=0.5, c=0.2$. Figure 1 (a) shows that $q_{1}^{O_{1}}$ is stable for all the values of $\nu_{1}$ until the parameter reaches the value $v_{1}=(2 / a)$ on where the period- 2 cycle arises. At $v_{1}=3.88$, Figure 1(b) presents the basins of attraction of the stable point $q_{1}^{O_{1}}$. It is also obvious that the basins are bounded by the box defined by $\left[0, q_{1}^{O_{-1}}\right] \times\left[0, q_{1}^{O_{-1}}\right]$. At $k=5.9$, we give a situation of unstable $q_{1}$ due to a chaotic attractor behavior. As shown in Figure 1(c), the basins of this chaotic attractor lie within the box $\left[0, q_{1}^{O_{-1}}\right] \times\left[0, q_{1}^{O_{-1}}\right]$. For the second part of (22), we have the same discussions. Figure 1(d) shows that $q_{2}{ }^{O_{2}}$ is stable for the values of $v_{2}$ until the parameter reaches $(2 / a)$. Reaching this value gives rise to periodic cycle and chaotic attractor. For instance, at the parameters set $a=0.5, \omega=0.9, c=0.2$, and $v_{2}=3.88$, the basins of attraction of the stable point $q_{2}^{O_{2}}$ are given in Figure 1(e). As $v_{2}$ increases to 5.9 , the point $q_{2}^{\mathrm{O}_{2}}$ gets unstable and chaotic attractor appears. The basins for this chaotic attractor are given in Figure 1(f). We can conclude that as $v_{2}$ increases further, any dynamic behavior will be bounded by the box $\left[0, q_{2}^{O_{-1}}\right] \times\left[0, q_{2}^{O_{-1}}\right]$.

Now, we carry out some numerical simulations in order to investigate and analyze the influences of the parameters $v_{1}$ and $v_{2}$ on the map given in (8). All numerical simulations in this section are performed at the initial datum $\left(q_{1}(0)\right.$, $\left.q_{2}(0)\right)=(0.11,0.12)$. Assuming the parameters set, $a=$ $0.5, c=0.2, d=0.35, \quad$ and $\omega=0.45$. This gives
$E_{*}=(0.1853171775,0.2637205988)$. Assuming $\nu_{1}=3.5$ and $v_{2}=4.2$, then (18) becomes

$$
\mathbf{J}\left(E_{*}\right) \approx\left(\begin{array}{rr}
-0.42694 & -0.22701 \\
-0.38767 & -0.82758
\end{array}\right),
$$

which has two real eigenvalues, $\lambda_{1} \approx-0.26930$ and $\lambda_{2} \approx-0.98522$. One can see that $\left|\lambda_{1,2}\right|<1$ and hence $E_{*}$ is a local stable point. Keeping the parameter set including $\nu_{2}$ fixed and increasing $\nu_{1}$ to 3.65 , the Jacobian $\mathbf{J}\left(E_{*}\right)$ gets

$$
\mathrm{J}\left(E_{*}\right) \approx\left(\begin{array}{rr}
-0.48810 & -0.23674 \\
-0.38767 & -0.82758
\end{array}\right),
$$

and then the real eigenvalues become $\lambda_{1} \approx-0.31058$ and $\lambda_{2} \approx-1.0051$. This means that $E_{*}$ is changed into an unstable saddle point. Now, we perform some numerical simulation experiments in order to get more insights on the dynamic of map (8) around the equilibrium point $E_{*}$. We start our analysis by investigating the effects of the adjustment parameters $v_{1}$ and $v_{2}$ on the map. In Figures 2(a) and 2(b), we present the bifurcation diagram for the influences of the parameters $v_{1}$ and $v_{2}$ on the quantities $q_{1}$ and $q_{2}$ at the parameters values, $a=0.5, c=0.2, d=0.35$, and $\omega=0.45$. They show that the equilibrium point may be destabilized due to period-doubling bifurcation. Figure 2(c) confirms the chaotic behavior of the map by presenting the largest Lyapunov exponent. In Figures 2(d)-2(h), we give some different dynamic situations of the map due to varying the parameter $v_{1}$ and keeping the parameter $v_{2}=4.2$. They present the attractive basins of periodic cycles 2, 4, and 8 . Besides that, we show in Figures 2(g) and 2(h) two different chaotic attractors for the map around the equilibrium point. We have two disconnected attractors around the equilibrium point given in Figure $2(\mathrm{~g})$ which gather together to form a one chaotic attractor as given in Figure 2(h).

Now, we study another situation when the parameter $v_{2}$ is varied and $\nu_{1}$ becomes constant at the value 5. Figure 3(a) shows the $1 \mathrm{D}$ bifurcation diagram taking $\nu_{1}$ as the bifurcation parameter and the other parameters are selected to be $a=0.5, c=0.2, d=0.35, \omega=0.45, \quad$ and $\quad v_{2}=5 \quad$ while Figure 3(b)) depicts the bifurcation diagram with respect to the parameter $v_{2}$ and the other parameters' values are $a=0.5, c=0.2, d=0.35, \omega=0.45$, and $v_{1}=3$. It is clear that the equilibrium point becomes locally asymptotically stable till it reaches the point on where it can be destabilized due to period-doubling bifurcation. Due to a series of perioddoubling bifurcated points, the map becomes chaotic and enters the chaos region and this is confirmed in Figure 3(c) which shows the Lyapunov exponent with respect to the variables $v_{1}$ and $v_{2}$. Now, we use some numerical experiments to investigate more the dynamics of the map. Setting the parameters' values to $a=0.5, c=0.2, d=0.35, \omega=0.45, \nu_{1}=5$, and $\nu_{2}=5$, we get in Figure 3(d)) four closed invariant sets around the equilibrium point. Further increase in $v_{2}$ to 5.2 makes these four sets convert into four disconnected chaotic attractors as shown in Figure 3(e) which turn into two chaotic attractors as $v_{2}$ increases to 5.3 as given in Figure 3(f). At $v_{2}=5.4$, a 


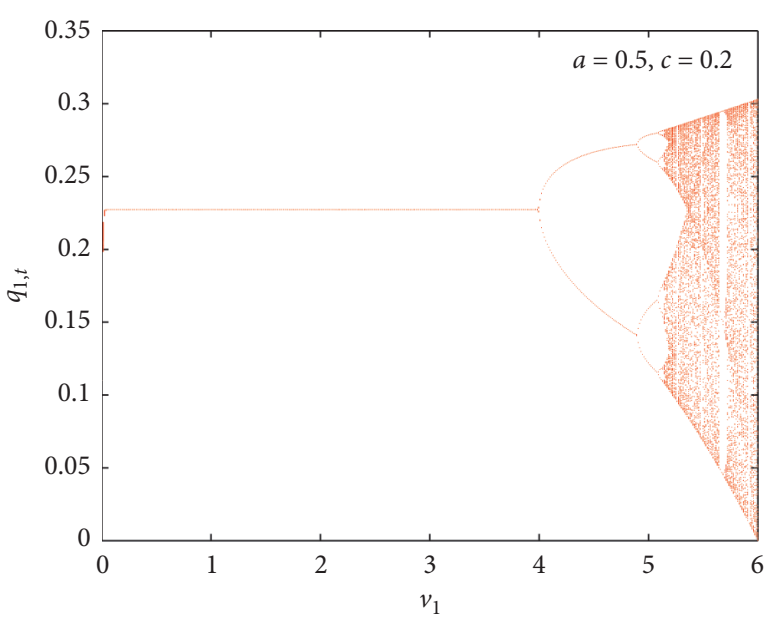

(a)

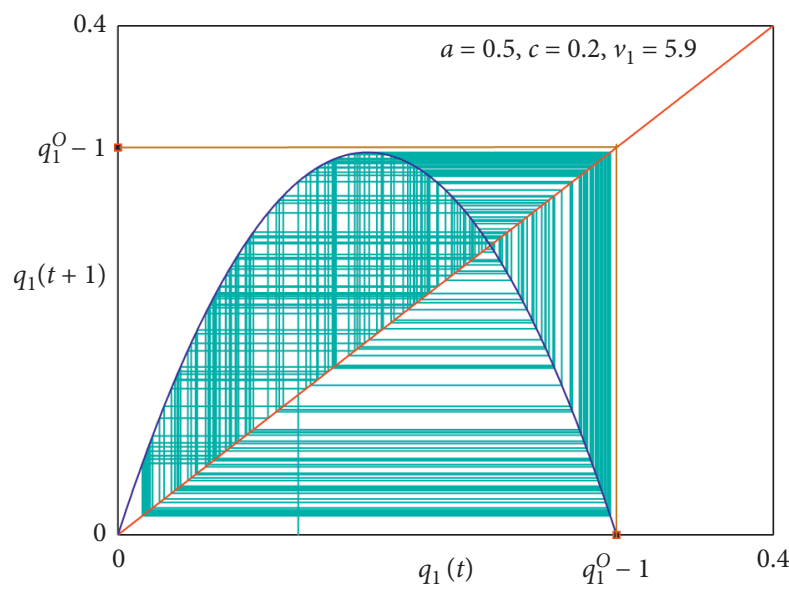

(c)

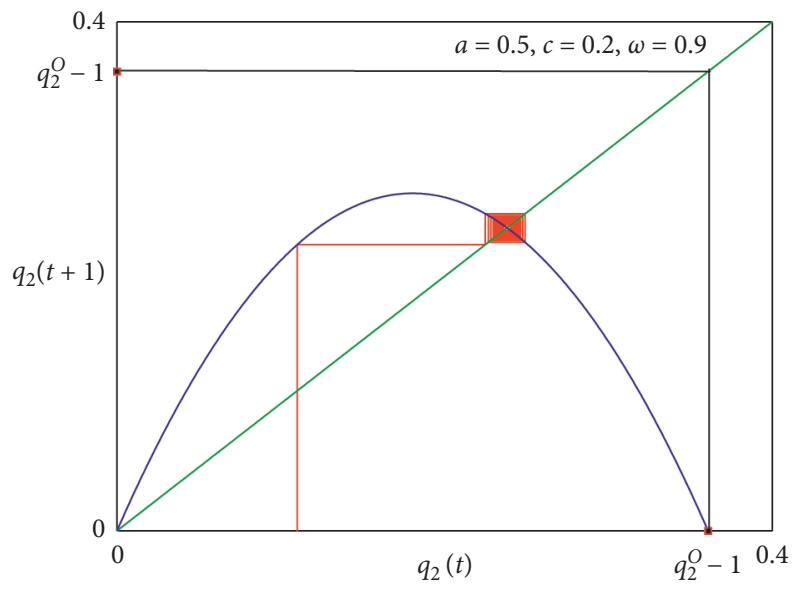

(e)

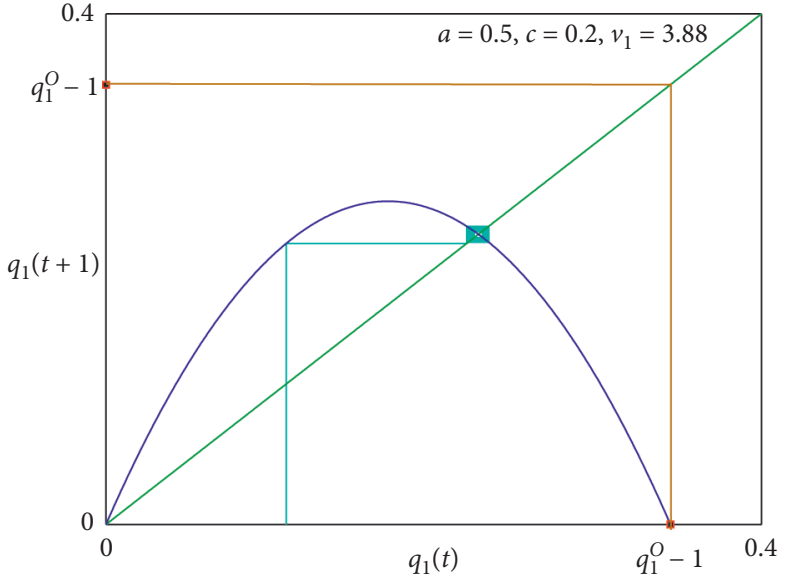

(b)

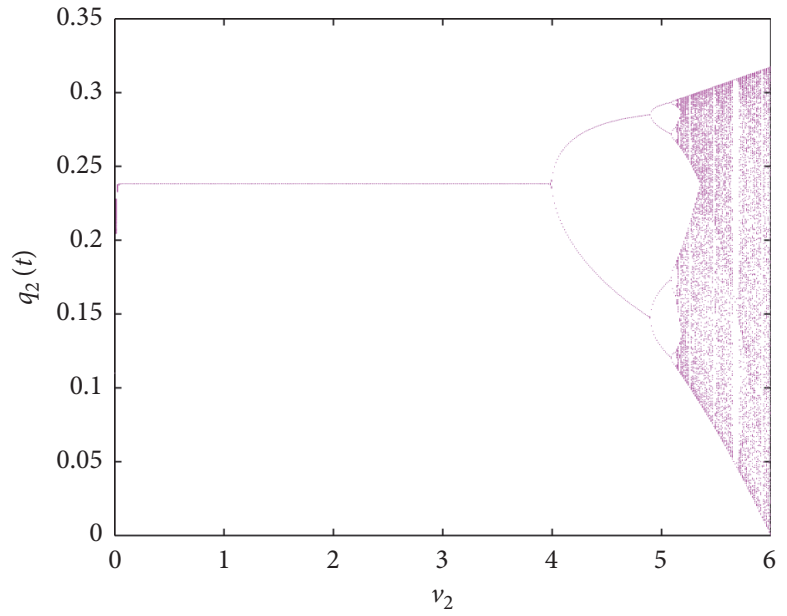

(d)

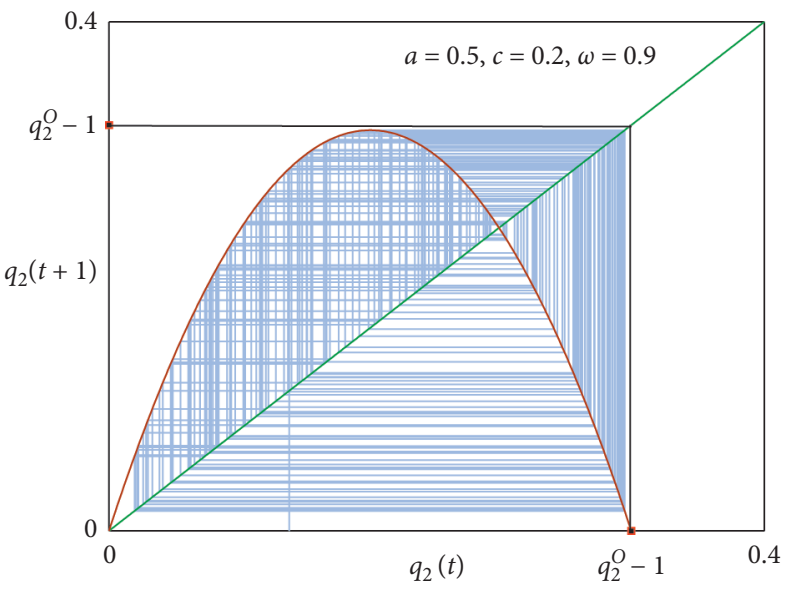

(f)

Figure 1: (a) Bifurcation diagram in $\left(\nu_{1}, q_{1}\right)$ - plane. (b) The basins of attraction of the stable point $q_{1}^{O_{1}}$ at $k=3.88$. (c) The basins of attraction of the stable point $q_{1}^{O_{1}}$ at $k=5.9$. (d) Bifurcation diagram in $\left(v_{2}, q_{2}\right)$ - plane. (e) The basins of attraction of the stable point $q_{2}^{\mathrm{O}_{2}}$ at $k=3.88$. (f) The basins of attraction of the stable point $q_{2}^{O_{2}}$ at $k=5.9$. The other values of the parameters are $a=0.5, c=0.2$, and $\omega=0.9$. 


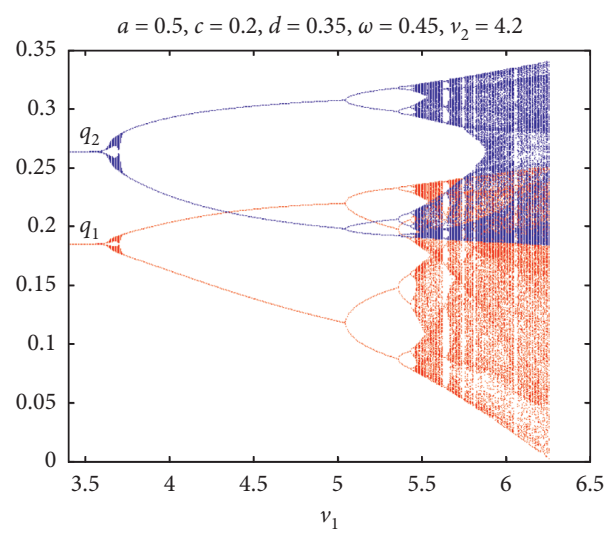

(a)
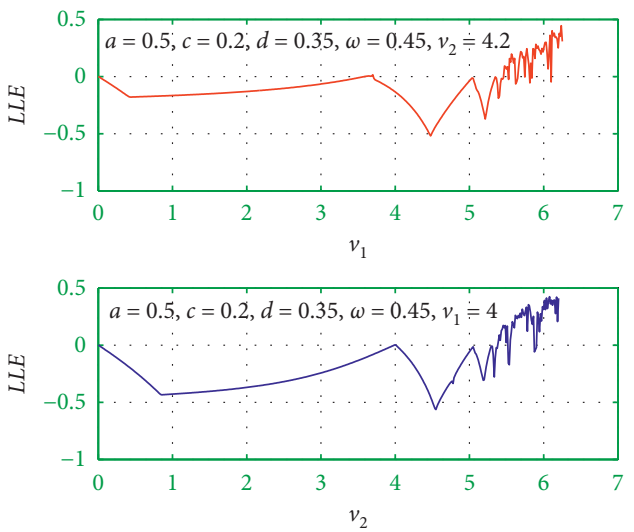

(c)

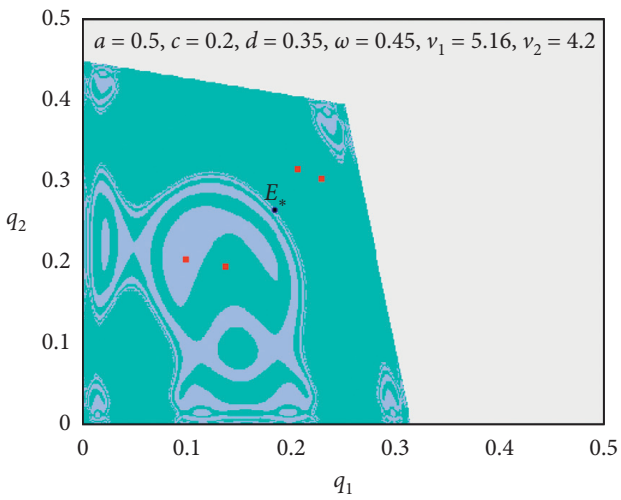

(e)

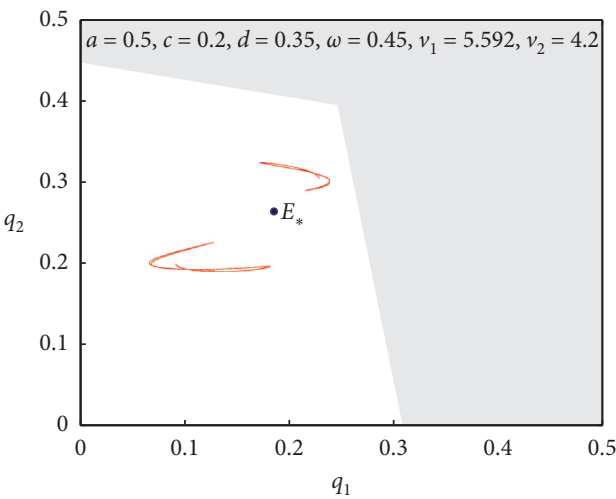

(g)

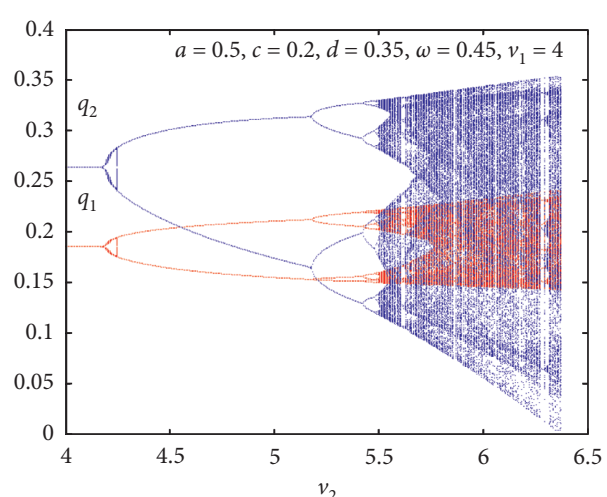

(b)

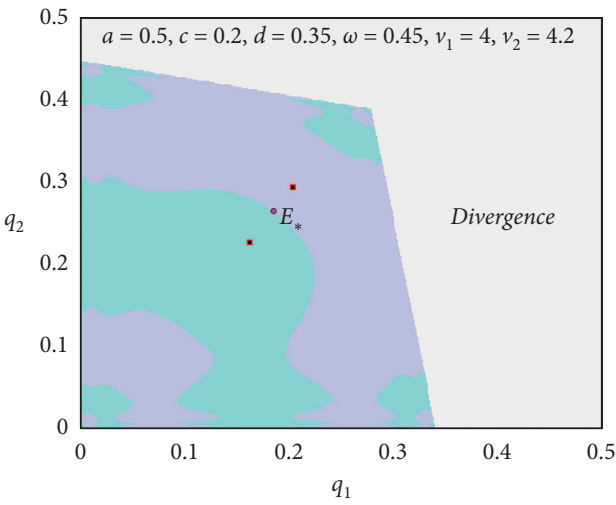

(d)

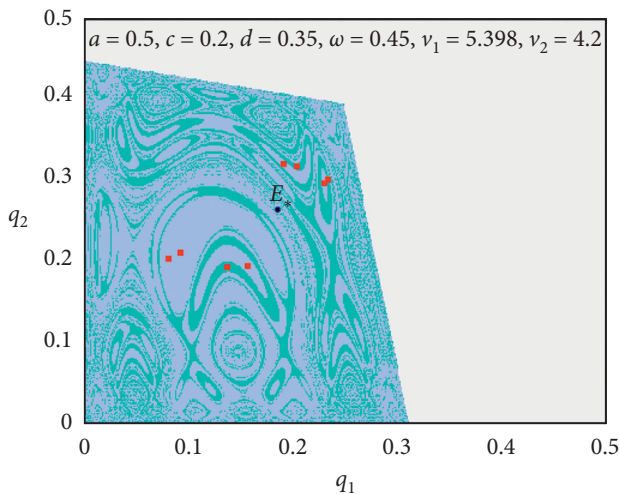

(f)

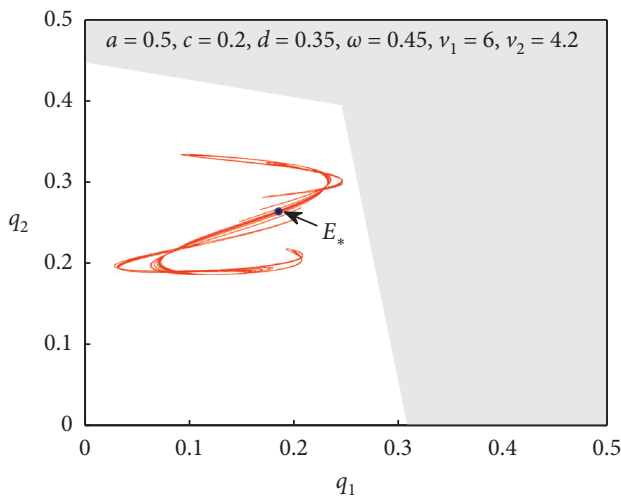

(h)

FiguRE 2: (a),(b) Bifurcation diagram with respect to $q_{1}$ and $q_{2}$ on varying $v_{1}$ and $v_{2}$. (c) Largest Lyapunov exponents with respect to $v_{1}$ and $\nu_{2}$. Basins of attraction for (d) period-2 cycle at $\nu_{1}=4$ and $\nu_{2}=4.2$. (e) Period-4 cycle at $\nu_{1}=5.16$ and $\nu_{2}=4.2$. (f) Period-8 cycle at $v_{1}=5.398$ and $v_{2}=4.2$. (g) Two unconnected chaotic attractors at $\nu_{1}=5.592$ and $\nu_{2}=4.2$. (h) One chaotic attractor at $v_{1}=6$ and $\nu_{2}=4.2$. Other parameters are $a=0.5, c=0.2, d=0.35$, and $\omega=0.45$. 


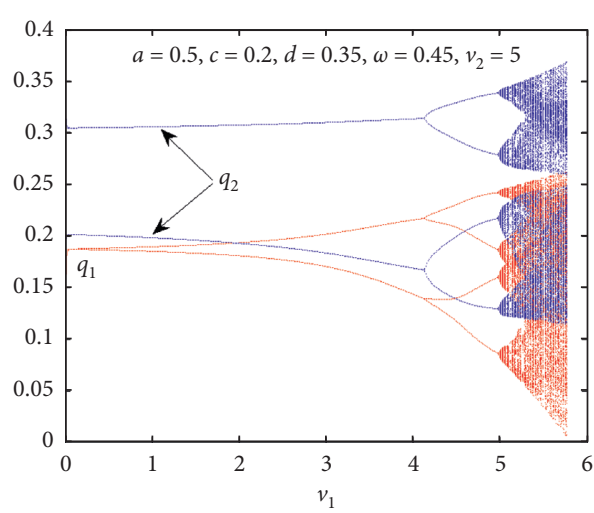

(a)
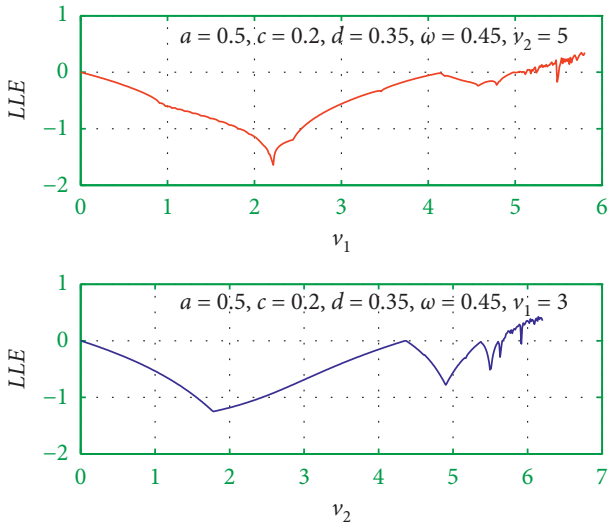

(c)

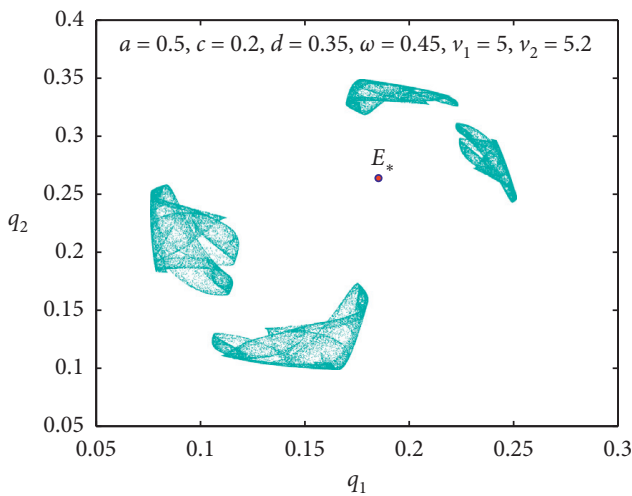

(e)

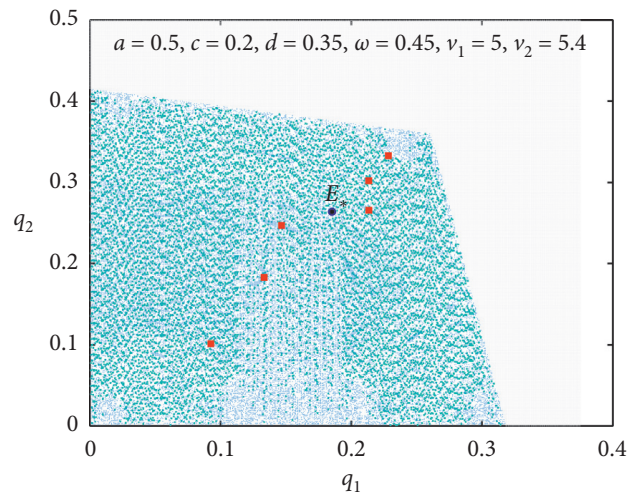

(g)

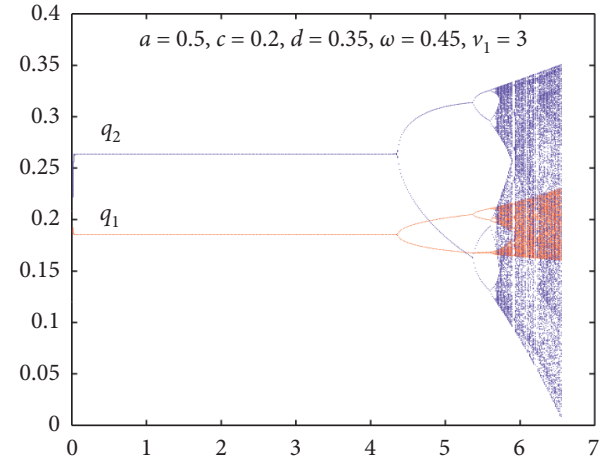

(b)

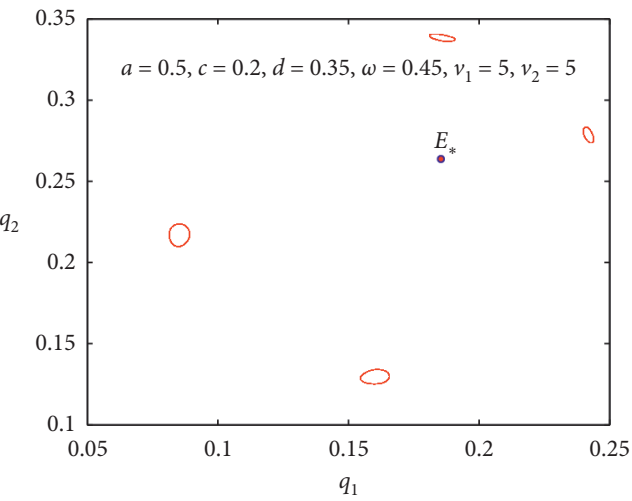

(d)

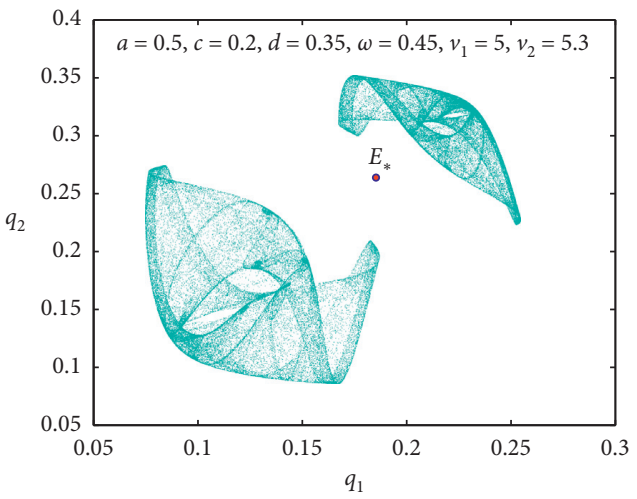

(f)

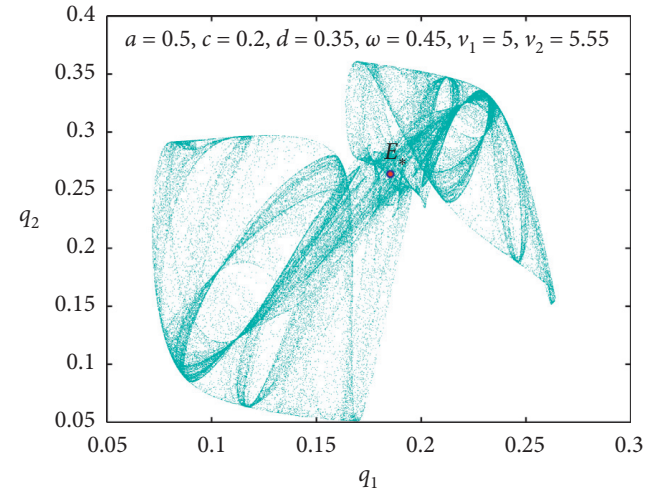

(h)

Figure 3: (a),(b) Bifurcation diagram with respect to $q_{1}$ and $q_{2}$ on varying $v_{1}$ and $v_{2}$. (c) Largest Lyapunov exponents with respect to $v_{1}$ and $\nu_{2}$. Phase plane for (d) four closed invariant sets at $\nu_{1}=5$ and $\nu_{2}=5$. (e) Four chaotic areas at $\nu_{1}=5$ and $\nu_{2}=5.2$. (f) Two chaotic areas at $\nu_{1}=5$ and $v_{2}=5.3$. (g) Basins of attraction of period -6 cycle at $v_{1}=5$ and $v_{2}=5.4$. (h) Phase plane for one chaotic attractor at $v_{1}=5$ and $v_{2}=5.55$. Other parameters are $a=0.5, c=0.2, d=0.35$, and $\omega=0.45$. 


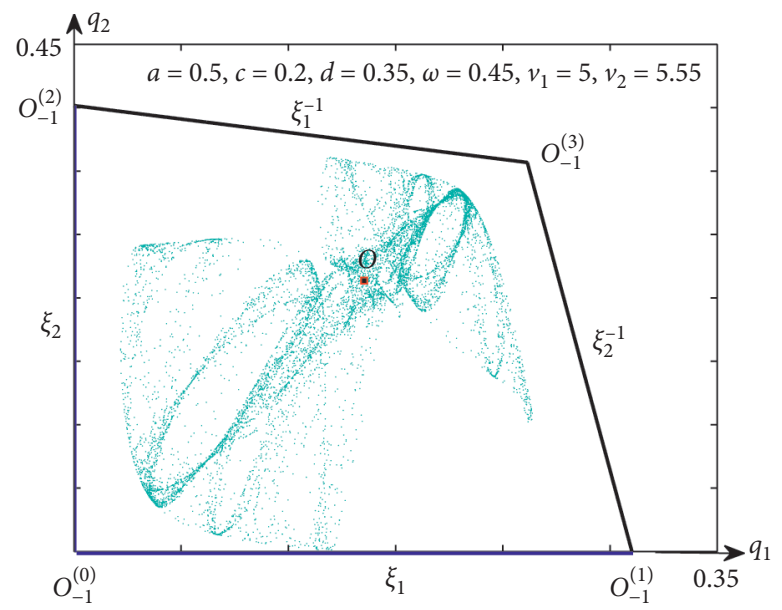

Figure 4: The boundaries of the chaotic attractor given in Figure 3(h).

period- 6 cycle is emerged and plotted in Figure 3(g) with its attractive basins. Increasing $\nu_{2}$ to 5.55 , the dynamics of the map become chaotic as depicted in Figure 3(h).

3.2. Noninvertible Map. We have previously discussed that the map $T$ is being trapped in the point $(0,0)$. This means that at $q_{1}(t)=0$ or $q_{2}(t)=0$, we get $q_{1}(t+1)=0$ or $q_{2}(t+1)=0$, respectively. So, the point $(0,0)$ is used to calculate basins' boundaries for any attracting set of the map. Doing that requires setting $q_{1}(t+1)=q_{1}$ and $q_{2}(t+1)=q_{2}$ in (8) as follows:

$$
T:\left\{\begin{array}{l}
\dot{q}_{1}=q_{1}(t)+v_{1} q_{1}\left[a-(2+c) q_{1}-\mathrm{d} q_{2}\right], \\
q_{2}=q_{2}(t)+v_{2} q_{2}\left[a-(1+\omega+c) q_{2}-\mathrm{d} q_{1}\right],
\end{array}\right.
$$

where, 1 indicates evolution of time. For map (29), if $T^{-1}:\left(q_{1}, q_{2}\right) \longrightarrow\left(q_{1}, q_{2}\right)$ gets unique value for each point in the range, then we call $T$ an invertible map and then the point $\left(q_{1}, q_{2}\right) \in \mathbb{R}^{2}$ is a rank-1 image while $\left(q_{1}, q_{2}\right)$ is a rank1 preimage., If there exist at least two rank-1 preimages for an image $\left(q_{1}, q_{2}\right)$, then the map $T$ is called a noninvertible map. Now, we calculate the real rank-1 preimages for the point $(0,0)$.

Proposition 4. The point $O=(0,0)$ possesses four real rank1 preimages,

Proof. Setting ' $q_{1}=0$ and $\dot{q}_{2}=0$ in (29) and solving algebraically, we get

$$
\begin{aligned}
& O_{-1}^{(0)}=(0,0), \\
& O_{-1}^{(1)}=\left(\frac{1+a v_{1}}{v_{1}(2+c)}, 0\right), \\
& O_{-1}^{(2)}=\left(0, \frac{1+a \nu_{2}}{v_{2}(1+c+\omega)}\right), \\
& O_{-1}^{(3)}=\left(\frac{a v_{1} \nu_{2}(1+c+\omega-d)+(1+c+\omega) \nu_{2}-d \nu_{1}}{v_{1} \nu_{2}\left(2+2 \omega+3 c+c \omega+c^{2}-d^{2}\right)}, \frac{a v_{1} \nu_{2}(2+c-d)+(2+c) \nu_{1}-d v_{2}}{v_{1} v_{2}\left(2+2 \omega+3 c+c \omega+c^{2}-d^{2}\right)}\right) .
\end{aligned}
$$

This completes the proof.

The above proposition indicates that any attracting set for map (27) has an attractive basin that is bounded by a quadrilateral shape whose boundaries are defined by the line segments $\xi_{1}=\mathrm{OO}_{-1}^{(1)}, \xi_{2}=\mathrm{OO}_{-1}^{(2)}$ and their preimages $\xi_{1}^{-1}$ and $\xi_{2}^{-1}$, respectively. These preimages are given by

$$
\begin{array}{r}
\xi_{1}^{-1}: q_{2}=\frac{1+a v_{2}-d v_{2} q_{1}}{v_{2}(1+c+\omega)}, \\
\xi_{2}^{-1}: q_{2}=\frac{1+a v_{1}-v_{1}(2+c) q_{1}}{d v_{1}} .
\end{array}
$$

Figure 4 displays those line segments and their preimages at the parameters' values: $a=0.5, c=0.2, d=$ $0.35, \omega=0.45, v_{1}=5$, and $v_{2}=5.55$. 


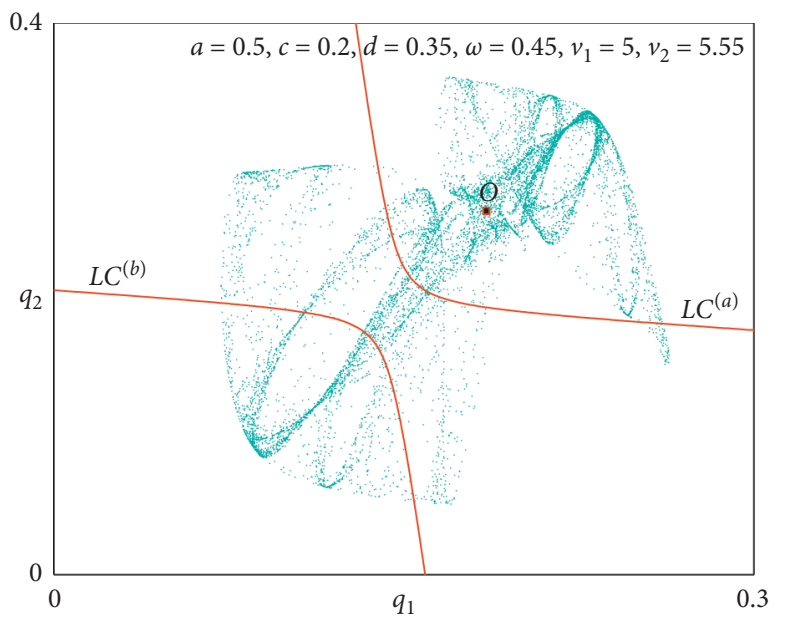

(a)

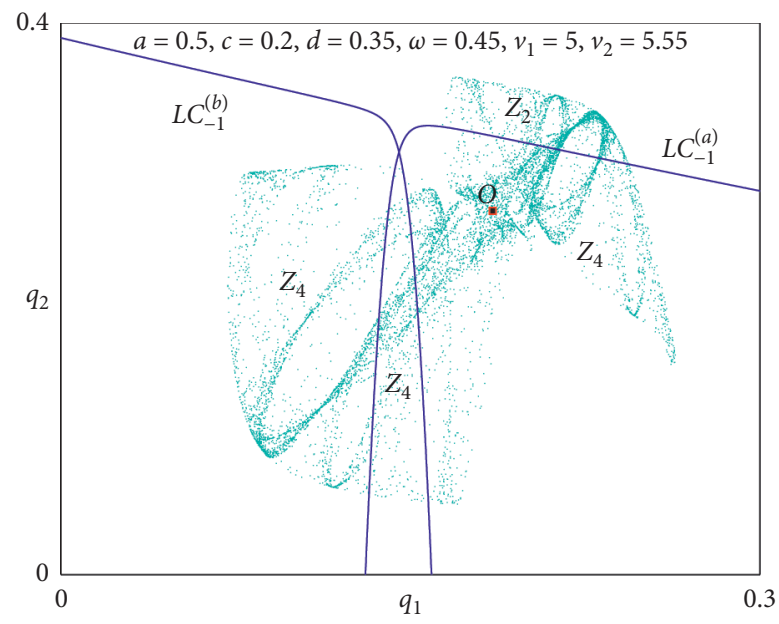

(b)

Figure 5: (a), (b) The critical curves LC, $\mathrm{LC}_{-1}$ and the region, $Z_{i}, i=2,4$ at the parameters' values: $a=0.5, c=0.2, d=0.35, \omega=0.45, v_{1}=5$, and $v_{2}=5.55$.

In Figure 5, we plot the critical curves $\mathrm{LC}$ and $\mathrm{LC}_{-1}$ at the parameters set, $a=0.5, c=0.2, d=0.35, \omega=0.45, v_{1}=5$, and $v_{2}=5.55$. It is clear that LC divides the phase plane into the two regions $Z_{4}$ and $Z_{2}$ as shown in Figures 5(a) and 5(b). Therefore, the map is noninvertible. In addition, the points $(0,0)$ and the equilibrium point $O$ are belonging to the region $Z_{4}$.

\section{Conclusion}

The current paper has studied a two-dimensional map that has described the competition between two Cournot firms one of which has considered the maximization of social welfare instead of profit. Based on a gradient-based mechanism, the map has been modelled in discrete time steps. It has been analyzed that when the two firms have become monopolistic, their dynamics have turned into just like the standard coupled logistic map. While in the duopolistic case, the map's fixed points have been calculated and their stability conditions have been investigated showing that the Nash equilibrium point may be unstable due to two types of bifurcations. Through some numerical analysis, we have shown some attracting sets with their basins of attraction and other chaotic behaviors of the map around the equilibrium point have been detected. The critical curves of the game's map have been calculated, and the corresponding preimages regions have been identified. We have shown that the map is noninvertible and has belonged to $Z_{4}-Z_{2}$ type. For future studies, we plan to investigate more such adoption of maximization of social welfare on heterogeneous players (or firms). We have shown that the model parameters, in particular the speed of adjustment and the degree of privatization of the second firm, have an effect on the long-term dynamic response of the game, which is important for understanding the functioning of the mixed duopoly game. This result allows players to gain a specific understanding of the mixed oligopoly market and to recognize that the choice of decision criteria would have a certain effect on the system's actions.

\section{Data Availability}

The datasets generated during the current study are available from the corresponding author on reasonable request.

\section{Conflicts of Interest}

The authors declare that they have no conflicts of interest.

\section{Acknowledgments}

This work was supported by King Saud University, Riyadh, Saudi Arabia (research-supporting project number RSP2020/167).

\section{References}

[1] A. A. Cournot, "Researches into the principles of the theory of wealth," in Classics in EconomicsAugustus M Kelley Pubs., New York, NY, USA, 1971.

[2] J. Bertrand, "Theorie mathematique de la richesse sociale," Journal des Savants, vol. 67, pp. 499-508, 1883.

[3] N. Singh and X. Vives, "Price and quantity competition in a differentiated duopoly," The RAND Journal of Economics, vol. 15, no. 4, pp. 546-554, 1984.

[4] S. S. Askar, "The Influences of asymmetric market information on the dynamics of duopoly game," Mathematics, vol. 8, no. 7, p. 1132, 2020.

[5] S. S. Askar and A. Al-Khedhairi, "Dynamic investigations in a duopoly game with price competition based on relative profit and profit maximization," Journal of Computational and Applied Mathematics, vol. 367, Article ID 112464, 2020.

[6] S. S. Askar, "Duopolistic Stackelberg game: investigation of complex dynamics and chaos control," Operational Research, vol. 20, no. 3, pp. 1685-1699, 2020.

[7] S. S. Askar and A. Al-Khedhairi, "The dynamics of a business game: a 2D-piecewise smooth nonlinear map," Physica A: Statistical Mechanics and Its Applications, vol. 537, Article ID 122766, 2020.

[8] A. A. Elsadany, "Dynamics of a Cournot duopoly game with bounded rationality based on relative profit maximization," 
Applied Mathematics and Computation, vol. 294, pp. 253-263, 2017.

[9] A. K. Naimzada and F. Tramontana, "Dynamic properties of a Cournot-Bertrand duopoly game with differentiated products," Economic Modelling, vol. 29, no. 4, pp. 1436-1439, 2012.

[10] J. Ma and X. Pu, "The research on Cournot-Bertrand duopoly model with heterogeneous goods and its complex characteristics," Nonlinear Dynamics, vol. 72, no. 4, pp. 895-903, 2013.

[11] T. Puu, "Chaos in duopoly pricing," Chaos, Solitons \& Fractals, vol. 1, no. 6, pp. 573-581, 1991.

[12] J. X. Zhang, D. Q. Zhou, and Y. H. Wang, "Complex dynamics analysis for a Bertrand duopoly model with bounded rationality," in Proceedings of the 2009 International Conference on Management Science and Engineering, pp. 190-195, IEEE, Moscow, Russia, September 2009.

[13] T. Puu, "The chaotic monopolist," Chaos, Solitons \& Fractals, vol. 5, no. 1, pp. 35-44, 1995.

[14] J. Long and H. Huang, "A dynamic stackelberg-cournot duopoly model with heterogeneous strategies through oneway spillovers," Discrete Dynamics in Nature and Society, vol. 2020, Article ID 3251609, 11 pages, 2020.

[15] H. N. Agiza and A. A. Elsadany, "Nonlinear dynamics in the Cournot duopoly game with heterogeneous players," Physica A: Statistical Mechanics and Its Applications, vol. 320, pp. 512-524, 2003.

[16] M. Kopel, "Simple and complex adjustment dynamics in cournot duopoly models," Chaos, Solitons \& Fractals, vol. 7, no. 12, pp. 2031-2048, 1996.

[17] E. M. Elabbasy, H. N. Agiza, A. A. Elsadany, and H. ELMetwally, "The dynamics of triopoly game with heterogeneous players," International Journal of Nonlinear Science, vol. 3, no. 2, pp. 83-90, 2007.

[18] E. M. Elabbasy, H. N. Agiza, and A. A. Elsadany, "Analysis of nonlinear triopoly game with heterogeneous players," Computers \& Mathematics with Applications, vol. 57, no. 3, pp. $488-499,2009$.

[19] S. S. Askar and M. Abouhawwash, "Quantity and price competition in a differentiated triopoly: static and dynamic investigations," Nonlinear Dynamics, vol. 91, no. 3, pp. 1963-1975, 2018.

[20] C. Hommes, Behavioral Rationality and Heterogeneous Expectations in Complex Economic Systems, Cambridge University Press, Cambridge, EN, UK, 2013.

[21] V. J. Tremblay, C. H. Tremblay, and K. Isariyawongse, "Cournot and Bertrand competition when advertising rotates demand: the case of Honda and Scion," International Journal of the Economics of Business, vol. 20, no. 1, pp. 125-141, 2013.

[22] E. Ahmed, M. F. Elettreby, and A. S. Hegazi, "On Puu's incomplete information formulation for the standard and multi-team Bertrand game," Chaos, Solitons \& Fractals, vol. 30, no. 5, pp. 1180-1184, 2006.

[23] L. C. Baiardi and A. K. Naimzada, "An oligopoly model with rational and imitation rules," Mathematics and Computers in Simulation, vol. 156, pp. 254-278, 2019.

[24] L. Fanti, L. Gori, C. Mammana, and E. Michetti, "The dynamics of a Bertrand duopoly with differentiated products: synchronization, intermittency and global dynamics," Chaos, Solitons \& Fractals, vol. 52, pp. 73-86, 2013.

[25] A. A. Elsadany and A. M. Awad, "Dynamics and chaos control of a duopolistic Bertrand competitions under environmental taxes," Annals of Operations Research, vol. 274, no. 1-2, pp. 211-240, 2019.
[26] C. H. Tremblay and V. J. Tremblay, "Oligopoly games and the cournot-bertrand model: a survey," Journal of Economic Surveys, vol. 33, no. 5, pp. 1555-1577, 2019.

[27] S. S. Askar and A. Al-Khedhairi, "Analysis of nonlinear duopoly games with product differentiation: stability, global dynamics, and control," Discrete Dynamics in Nature and Society, vol. 11, p. 2017, 2017.

[28] B. Gao and Y. Du, "Equilibrium further studied for combined system of cournot and bertrand: a differential approach," Complexity, vol. 2020, Article ID 3160658, 11 pages, 2020.

[29] L. Liu, X. H. Wang, and C. Zeng, "Endogenous horizontal product differentiation in a mixed duopoly," Review of Industrial Organization, vol. 56, no. 3, pp. 435-462, 2020.

[30] Y. Liu, T. Matsumura, and C. Zeng, "The relationship between privatization and corporate taxation policies," Journal of Economics, vol. 132, 2021.

[31] P.-Y. Nie, "Effects of capacity constraints on mixed duopoly," Journal of Economics, vol. 112, no. 3, pp. 283-294, 2014.

[32] P. Y. Nie and Y. C. Yang, "Cost-reduction innovation under mixed economy," Managerial and Decision Economics, vol. 41, no. 7, p. 1195, 2020. 